\title{
Applications of deep learning to relativistic hydrodynamics
}

\author{
Hengfeng Huang, ${ }^{1,2}$ Bowen Xiao, ${ }^{3}$ Ziming Liu, ${ }^{1}$ Zeming Wu, ${ }^{1,2}$ Yadong Mu, ${ }^{3,4}$ and Huichao Song $\oplus^{1,2,5}$ \\ ${ }^{1}$ Department of Physics and State Key Laboratory of Nuclear Physics and Technology, Peking University, Beijing 100871, China \\ ${ }^{2}$ Collaborative Innovation Center of Quantum Matter, Beijing 100871, China \\ ${ }^{3}$ Institute of Computer Science and Technology, Peking University, Beijing 100080, China \\ ${ }^{4}$ Center for Data Science, Peking University, Beijing 100871, China \\ ${ }^{5}$ Center for High Energy Physics, Peking University, Beijing 100871, China
}

(Received 19 April 2018; accepted 2 April 2021; published 30 June 2021)

\begin{abstract}
Relativistic hydrodynamics is a powerful tool to simulate the evolution of the quark-gluon plasma in relativistic heavy-ion collisions. Using 10000 initial and final profiles generated from (2+1)-dimensional relativistic hydrodynamics VISH2+1 with Monte Carlo Glauber (MC-Glauber) initial conditions, we train a deep neural network based on the stacked U-net, and use it to predict the final profiles associated with various initial conditions, including MC-Glauber, MC Kharzeev-Levin-Nardi (MC-KLN), a multiphase transport (AMPT) model, and the reduced thickness event-by-event nuclear topology (TRENTo) model. A comparison with the VISH $2+1$ results shows that the network predictions can nicely capture the magnitude and inhomogeneous structures of the final profiles, and creditably describe the related eccentricity distributions $P\left(\varepsilon_{n}\right)(n=2,3,4)$. These results indicate that a deep learning technique can capture the main features of the nonlinear evolution of hydrodynamics, showing its potential to largely accelerate the event-by-event simulations of relativistic hydrodynamics.
\end{abstract}

DOI: 10.1103/PhysRevResearch.3.023256

\section{INTRODUCTION}

In recent years deep learning [1-3] has achieved great success in both daily life and in sciences. In particular, deep learning methods have been implemented in various research areas in physics, including the search for gravitational lenses [4,5], identifying and classifying the phases of the Ising model [6-9], solving the quantum many-body problem [10,11], etc. In high-energy physics, it has been applied to the search for Higgs and exotic particles [12,13], the classification of jet structures [14-16], etc. In the field of relativistic heavy-ion collisions, machine learning and deep neural networks have been employed to attack the problems of identifying the equation of state (EOS) of hot QCD matter [17], jet-flavor classification in heavy-ion collisions [18], distinguishing between spinodal and Maxwell first-order phase transitions [19], detecting nuclear shape deformations [20], Bayesian extraction of transport properties of the hot QCD matter [21-24], the phase diagram of twodimensional complex scalar field theory [25], and principal component analyses of collective flow [26-32].

In this paper, we will apply deep learning to relativistic hydrodynamics, which is a useful tool to simulate the macroscopic evolution of relativistic systems in high-energy nuclear physics and astrophysics [33]. Relativistic hydrodynamics solves the transport equations of the energy momentum ten-

Published by the American Physical Society under the terms of the Creative Commons Attribution 4.0 International license. Further distribution of this work must maintain attribution to the author(s) and the published article's title, journal citation, and DOI. sor and charge currents based on the conservation laws. In relativistic heavy-ion collisions, it has nicely described and predicted various flow data of the quark-gluon plasma (QGP), which played an important role in the discovery of the strongly coupled QGP and its nearly perfect fluid nature [21-24,34-46]. However, traditional hydrodynamic simulations are time consuming. For example, the calculation of various flow harmonics requires $\sim 1000$ event-by-event hydrodynamic simulations, which takes $\sim 500$ and $\sim 10000 \mathrm{cpu}$ hours for the typical (2+1)-dimensional (2+1D) and (3+1)dimensional (3+1D) simulations, respectively [38-40,47]. Basically, relativistic hydrodynamics translates the initial conditions into final profiles through solving a set of nonlinear differential equations. In this work, we will explore whether the deep neural network could capture the main features of the nonlinear evolution of 2+1D hydrodynamics, and the possibilities to accelerate the related event-by-event simulations. Close to this work are Bayesian emulators [24,48], which are powerful in constraining the equation of states and transport coefficients, yet are not designed to predict the whole profiles of the energy density and flow velocity.

It is worthwhile to mention that the interdisciplinary contributions of this work are twofold: From the physics perspective, we speed up hydrodynamic simulations time with a deep neural network, while still capturing the details for the final profiles of the expanding QGP. On the other hand, from the machine learning angle, we highlight the expressive power of the stacked U-net model, as well as its ability to approximate the partial differential equation (PDE) in this particular task of relativistic hydrodynamics.

The paper is organized as follows: In Sec. II, we introduce the relativistic hydrodynamics and network design. In Sec. III, 

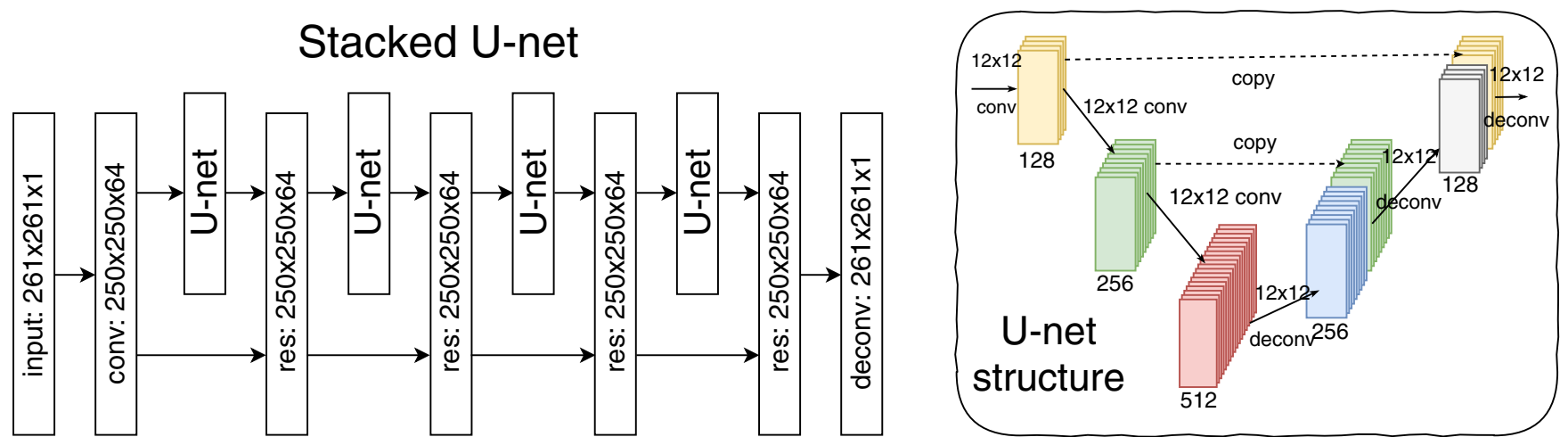

FIG. 1. An illustration of the encoder-decoder network, stacked U-net, which consists of the input convolutional layers and the output deconvolutional layers and four residual U-net blocks. The right figure shows the U-net structure, and the depth of the hidden layer is written on the top of them.

we show the results obtained from the network, followed by discussions and conclusions in Sec. IV.

\section{MODELS}

\section{A. Relativistic hydrodynamics}

In this paper, we focus on relativistic ideal hydrodynamics with zero viscosity and charge densities, which solves the transport equations of the energy momentum tensor $T^{\mu \nu}$ :

$$
\partial_{\mu} T^{\mu \nu}=0
$$

where $T^{\mu \nu}=(e+p) u^{\mu} u^{\nu}-p g^{\mu \nu}, e$ is the energy density, $p$ is the pressure, and $u^{\mu}$ is the four-velocity with $u^{\mu} u_{\mu}=1$. With an assumption of longitudinal boost invariance, we solve the $2+1 \mathrm{D}$ hydrodynamic equations with an ideal EOS $p=\frac{e}{3}$, using the code VISH $2+1[47,49]$ [50]. The initial energy density profiles can be generated by some initial condition models, such as the Monte Carlo Glauber (MC-Glauber) [51,52], MC Kharzeev-Levin-Nardi (MC-KLN) [52-54], a multiphase transport (AMPT) [55-57], and the reduced thickness eventby-event nuclear topology (TRENTo) [58] with zero initial transverse flow velocity. We run VISH $2+1$ with three selected fixed evolution times $\tau-\tau_{0}=2.0,4.0$, and $6.0 \mathrm{fm} / c\left(\tau_{0}=\right.$ $0.6 \mathrm{fm} / c)$ to obtain the energy momentum tensor $T^{\tau \tau}(\tau, x, y)$, $T^{\tau x}(\tau, x, y)$, and $T^{\tau y}(\tau, x, y)$ profiles at these times. For numerical accuracy, the time step and grid sizes of the simulations are set to $d \tau=0.04 \mathrm{fm} / c$ and $d x=d y=0.1 \mathrm{fm}$, within a fixed transverse area of $13 \mathrm{fm} \times 13 \mathrm{fm}$ that have been used to describe the typical QGP expansion in relativistic heavy-ion collisions.

\section{B. Network design}

For deep learning, the initial and final energy momentum tensor $T^{\tau \tau}, T^{\tau x}, T^{\tau y}$ profiles from hydrodynamics are treated as initial and final image sets with $261 \times 261$ pixels. In practice, we first run the event-by-event hydrodynamic simulations to obtain 10000 initial and final image sets, then use them to train the deep neural network, which aims at achieving nice predictions of the final energy density and flow velocity profiles for other input initial conditions.
The related network we adopted in this work is the stacked U-net (sU-net) [59], which is a variation of the traditional encoder-decoder network that could enhance gradient flow in the deeper part of the network during back propagation. Figure 1 presents an intuitional view of the network structure. It consists of four serially connected U-net blocks with residual connections between them. Each U-net block has three convolution layers and three deconvolution layers. In each U-net block, the outputs of the first two convolution layers are also fed into the last two deconvolution layers respectively by concatenating the feature maps along the channel dimension. The activation function for all layers except for the output one is Leaky ReLU $f(x)=\max \{x, 0.03 x\}$, while that for the output layer is softplus $f(x)=\ln \left(1+e^{x}\right)$ for $T^{\tau \tau}$ mapping and $f(x)=x$ for $T^{\tau x}$ and $T^{\tau y}$ mapping. To make the network focus more on local patterns, we set the kernel size of all convolution and deconvolution layers to $3 \times 3$. The loss function of the network is normalized mean absolute error (MAE) loss Loss $=\frac{\left|y_{1}-y_{0}\right|}{\left|y_{0}\right|}$, where $y_{1}$ is the output of the network and $y_{0}$ is the ground truth. We use the standard minibatch stochastic gradient descent algorithm for optimization. The batch size for training is 16 and the learning rate exponentially decays from $10^{-3}$ to $10^{-5}$. Each weight is randomly initialized from the uniform distribution on $[-0.001,0.001]$ and each bias is set to zero. Our code is built with TENSORFLOW and the training process runs for about 1 day on a machine with a single NVIDIA Tesla P40 graphics processing unit (GPU), using 10000 "initial" and "final" profiles from VISH2+1 hydrodynamic simulations.

We have noticed that, although one trained sU-net can make nice predictions for a shorter hydrodynamic evolution, it fails to accurately predict the final profiles of longer evolution times $\left(\tau-\tau_{0}>4.0 \mathrm{fm} / c\right)$ from the initial profiles at $\tau_{0}$. Considering that the evolving QGP system is highly nonlinear and tends to smear out its initial structures during a longer evolution, we divide the whole evolution time $\tau-\tau_{0}$ into $n$ parts with an equal time interval $\Delta \tau: \tau-\tau_{n-1} \cdots \tau_{2}-\tau_{1}, \tau_{1}-\tau_{0}$. For each evolution part, we train an individual sU-net using the corresponding initial and final profiles from hydrodynamics. To predict the final profiles at $\tau$ from initial profiles at $\tau_{0}$, we first use the trained sU-net- 1 to predict the profiles at time $\tau_{1}$ and then use them as the initial conditions for sU-net-2 


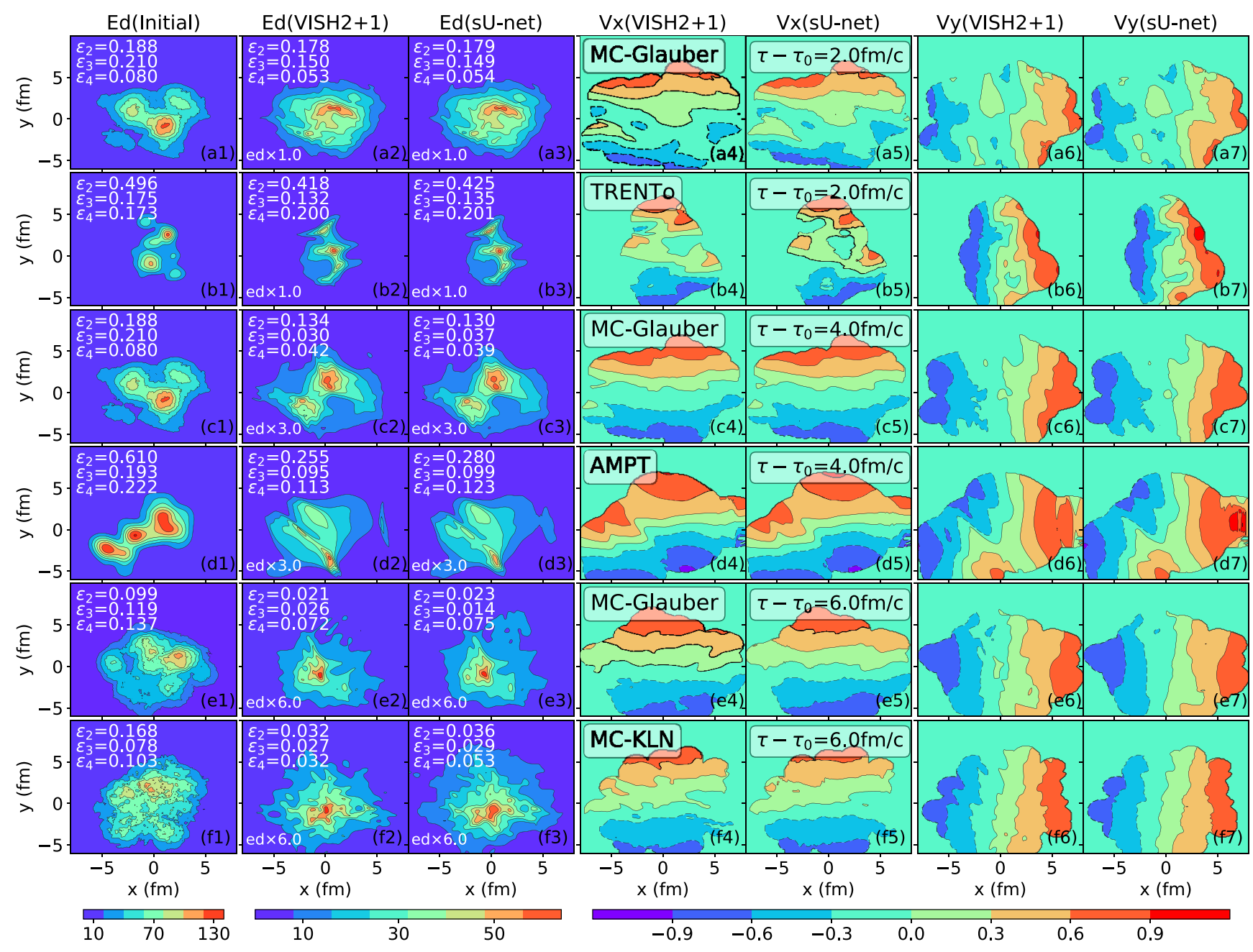

FIG. 2. Energy density and flow velocity profiles at $\tau-\tau_{0}=2.0,4.0$, and $6.0 \mathrm{fm} / c$, calculated from VISH $2+1$ and predicted by the network for six test cases with initial profiles generated from MC-Glauber, MC-KLN, AMPT, and TRENTo.

to predict the profiles at time $\tau_{2}$ and so on. In this way, the combined sU-net series $(i=1 \ldots n)$ mimic the hydrodynamic evolution with a much larger time step $\Delta \tau$ that cannot be managed by a traditional hydrodynamic algorithm (in more detail, for the following evolution with $\tau-\tau_{0}=6.0 \mathrm{fm} / c$, we set $n=3$ with $\Delta \tau=2.0 \mathrm{fm} / c$ ). Note that sU-net-1, sU-net-2, and sU-net-3 are not identical since the initial and final profiles are generated by VISH $2+1$, which implements the $2+1 \mathrm{D}$ hydrodynamic equations explicitly depended on $\tau[49,60]$.

\section{RESULTS}

As explained in the above text, we first use 10000 initial and final image sets from VISH2+1 with MC-Glauber initial conditions to train the combined stacked U-net, and then use the trained network to predict the final profiles from the initial profiles generated from MC-Glauber, MC-KLN, AMPT, and TRENTo as tests. Figure 2 presents a comparison between the results from VISH $2+1$ hydrodynamic evolution and the predictions from the network at $\tau-\tau_{0}=2.0,4.0$, and $6.0 \mathrm{fm} / c$ for six selected test cases. It shows that a well-designed and trained network could nicely predict the final states, which captures the structures of the contour plots for both the fi- nal energy density and flow velocity. It is impressive that, although the network is trained with the initial and final image sets associated with the MC-Glauber initial conditions, it could still creditably predict the final profiles of other initial conditions with different fluctuation patterns, as shown in Figs. 2(b), 2(d) and 2(f).

To further evaluate the predictive power of the network, we further calculate the eccentricity coefficients

$$
\varepsilon_{n}=\frac{\int r d r d \phi r^{n} e(r, \phi) e^{i n \phi}}{\int r d r d \phi r^{n} e(r, \phi)} \quad(n=2,3,4)
$$

for the initial and final energy density $e(r, \phi)$ profiles, which are quantities commonly used to evaluate the deformation and inhomogeneity of the QGP fireball in relativistic heavy-ion collisions [38-40,47]. These values of $\varepsilon_{n}(n=2,3,4)$ for these six selected test cases are written in Figs. 2(a)-2(f). From Fig. 2 and the calculated values of $\varepsilon_{n}(n=2,3,4)$, we have also noticed that differences between the hydrodynamic results and the network predictions increase for a longer evolution time since the combined sU-net series tend to accumulate errors with more sU-net added. 


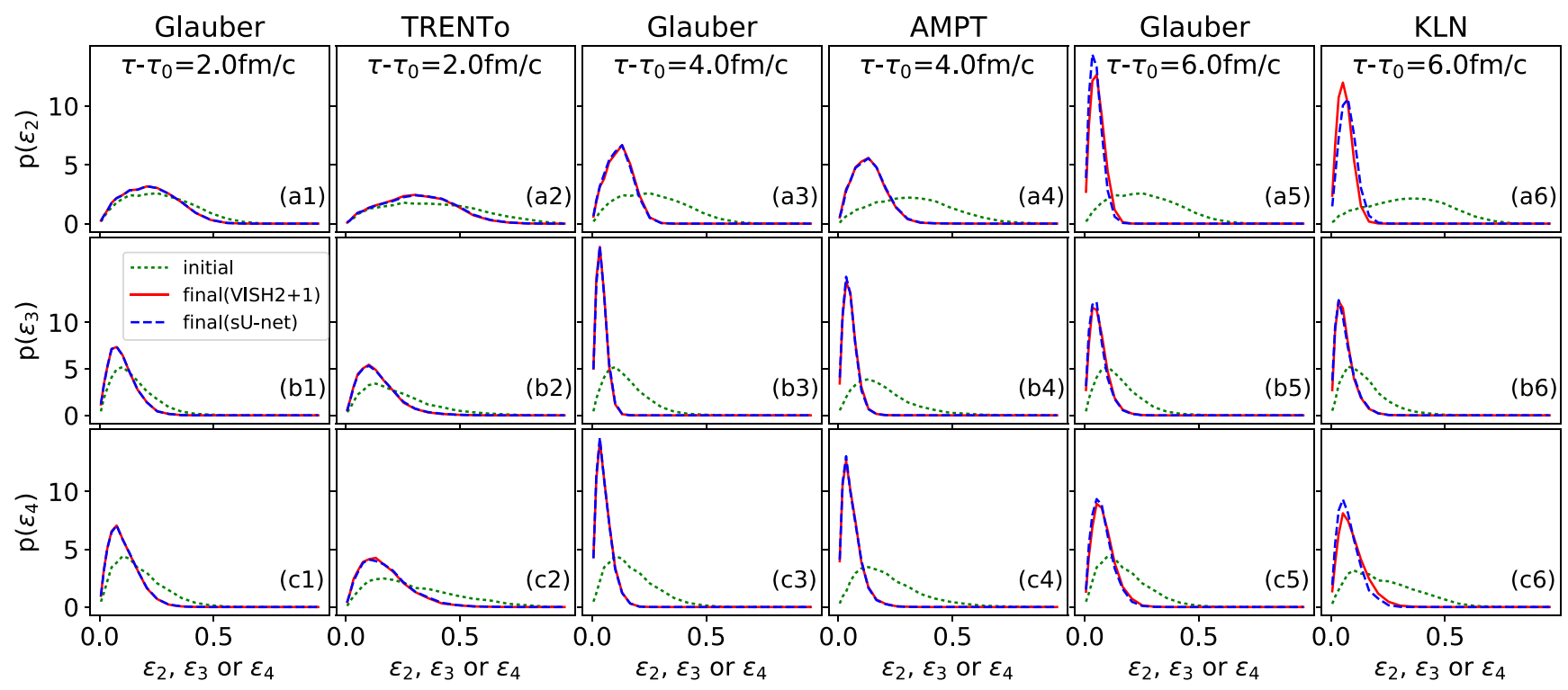

FIG. 3. Eccentricity distribution $P\left(\varepsilon_{n}\right)(n=2,3,4)$, at $\tau-\tau_{0}=2.0,4.0$, and $6.0 \mathrm{fm} / c$, calculated from VISH $2+1$ and predicted by the network for 10000 tested initial profiles generated from MC-Glauber, MC-KLN, AMPT, and TRENTo.

Figure 3 presents the eccentricity distributions $P\left(\varepsilon_{n}\right)$ for the energy density profiles at evolutions times $\tau-\tau_{0}=2.0$, 4.0 , and $6.0 \mathrm{fm} / c$, calculated from VISH $2+1$ and predicted from the network for 10000 tested initial profiles generated from MC-Glauber, MC-KLN, AMPT, and TRENTo. For all these tested cases, the final eccentricity distributions $P\left(\varepsilon_{n}\right)$ $(n=2,3,4)$ from the network almost overlap with the ones from VISH $2+1$, which also obviously deviate from the initial eccentricity distributions $P_{0}\left(\varepsilon_{n}\right)$. In Fig. 4, scatter plots show event-by-event comparisons between true eccentricities of the "final" profiles and predicted ones, and histograms of the errors are plotted in the inset figure.

We also find that, with the well-trained network, the final state profiles can be speedily generated from the initial profiles. Compared with the 10-20 min calculation time with a traditional CPU for a single-event hydrodynamic evolution, the network takes several seconds to directly generate the final profile for different types of initial profiles with the P40 GPU, which shows the potential to accelerate the realistic event-byevent hydrodynamic simulations in the near future. However, given the fact that a 50-100 $\times$ speedup of hydrodynamic simulations can be already achieved by switching from CPU to GPU [61,62], we believe there is still much room to improve our proof-of-concept first step in further studies.

\section{DISCUSSION AND CONCLUSION}

Using 10000 initial and final energy momentum tensor profiles from VISH2+1 hydrodynamics with MC-Glauber initial conditions, we successfully trained a deep neural network based on stacked U-net, and use it to predict the final profiles for different initial conditions, including MC-Glauber, MC-KLN, AMPT, and TRENTo. A comparison with the VISH2+1 results showed that the network predictions could nicely capture the magnitude and inhomogeneous structures of the final profiles, which also creditably describe the related eccentricity distributions $P\left(\varepsilon_{n}\right)(n=2,3,4)$. These results in- dicate that deep learning could capture the main feature of the nonlinear evolution of hydrodynamics, which also shows the potential of largely accelerating the realistic event-by-event hydrodynamic simulations in relativistic heavy-ion collisions.

In order to outline the highlights, as well as point out the limitations of this work, we further explain the following characteristics that mark good works and provide guidelines for future studies.

Universality. Deep learning might not learn the realistic physics underlying the data set. By contrast, sometimes its predictions are based on nonphysical features in the data set, as has been pointed out in Ref. [63]. In this work, we exclude such an undesirable possibility by training our deep model on MC-Glauber initial conditions and test on results for other initial models including MC-KLN, AMPT, and TRENTo.

Causality. Due to the speed of light as an upper bound for all physical speeds, our neural network should satisfy such causal relations, otherwise it will produce nonphysical results. The joint use of convolutional layers and the stacked structure elegantly handles this issue by allowing one pixel to influence its neighborhoods only. Such causality in convolutional layers is known as the receptive field [64] in the machine learning literature. More concretely, supposing our convolutional neural network $(\mathrm{CNN})$ has $L$ layers with the $i$ th layer using convolutional filters of size $\left(2 n_{i}+1\right) \times\left(2 n_{i}+1\right)$, then it is reasonable to match the size of the receptive field $R_{r}=\left(\sum_{i} n_{i}\right) \Delta x$ ( $\Delta x$ is the grid length) with the size of the light cone $R_{l}=c\left(\tau-\tau_{0}\right)$. If $R_{r}<R_{l}$, the expressivity of the neural network is bottlenecked. If $R_{r}<R_{l}$, the neural network is unnecessarily expressive, which might lead to a longer training time.

Utility. One limitation of this work is the fixed time output. Future works will consider more flexible architectures (e.g., a physics-informed neural network in Ref. [65]) to obtain the energy-momentum tensor at the freeze-out surface with a more realistic implementation in heavy-ion collisions. 


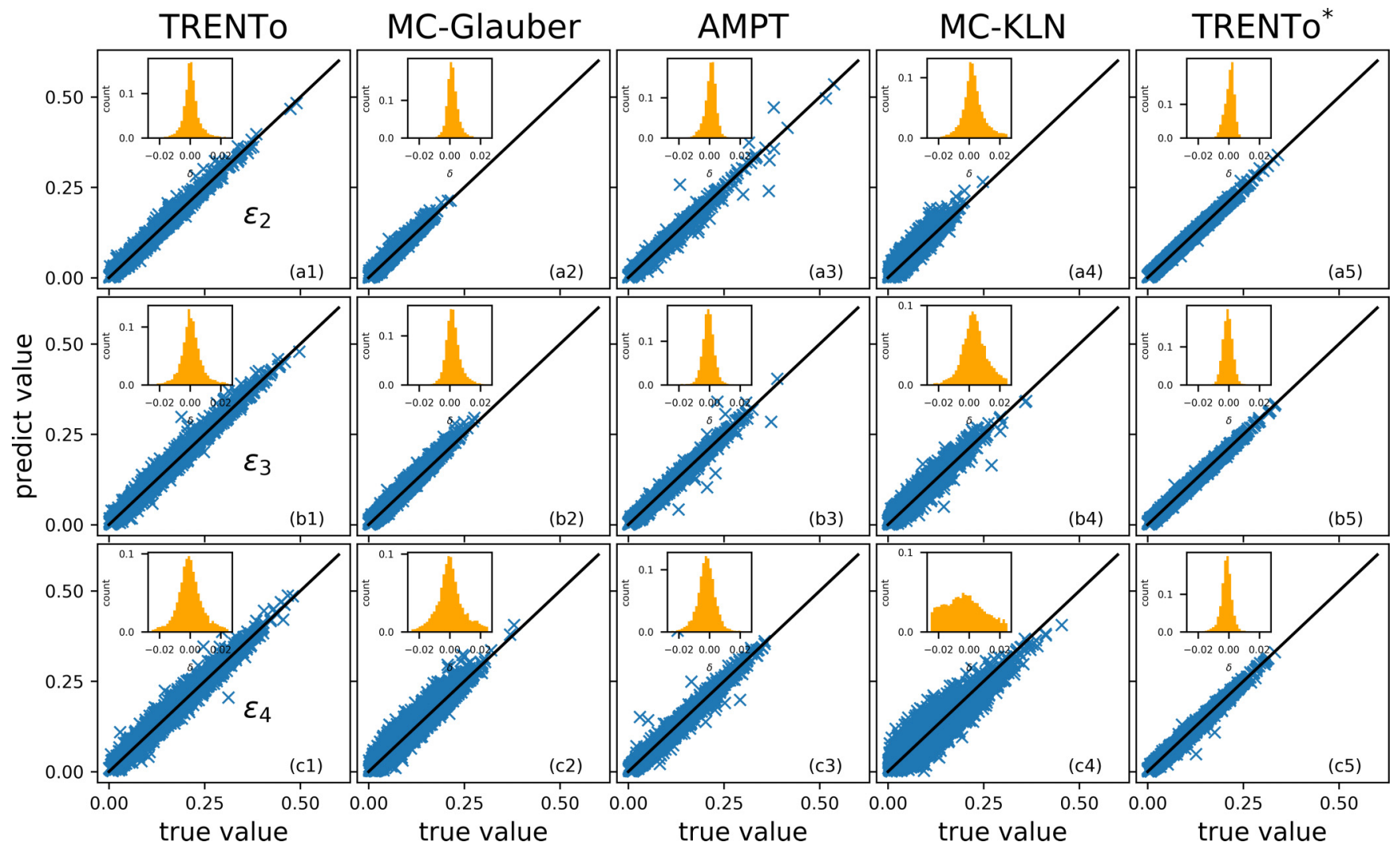

FIG. 4. Event-by-event comparisons of eccentricities $\varepsilon_{n}(n=2,3,4)$ at $\tau-\tau_{0}=6.0 \mathrm{fm} / c$, calculated from VISH $2+1$ and predicted by the network for 10000 tested initial profiles generated from MC-Glauber, AMPT, MC-KLN, and TRENTo (with two sets of parameters distinguished by $*$ ).

Interpretability. Another minor limitation of the stacked U-net model lies in the lack of interpretability. In future works, we will investigate the possibilities of encoding physics explicitly in the network design, as in Ref. [65]. Efforts on gaining interpretability of deep learning in heavy-ion collisions include Refs. [17,20].

In summary, our current investigations mainly focus on mimicking $(2+1)$-dimensional hydrodynamic evolution with a fixed evolution time, using the deep learning technique. On the one hand, for a more realistic implementation to relativistic heavy-ion collisions, it is worthwhile to explore the possibilities of mapping the initial profiles to the final profiles on the freeze-out surface with a fixed energy density as well as extending the related investigations to $(3+1)$-dimensional simulations. On the other hand, it is also worthwhile to develop computational tools that are more transparent for scientific evaluations, where a possible way is to encode the physical features (the functional form of the PDE, boundary conditions, etc.) into the network architecture.

\section{ACKNOWLEDGMENTS}

We are thankful for discussions with L. G. Pang, K. Zhou, and X.-N Wang. H.H., Z.L., and H.S. are supported by the NSFC under Grants No. 12075007 and No. 11675004. B.X. and Y.M. are supported by NSFC under Grant No. 61772037.
[1] I. Goodfellow, Y. Bengio, and A. Courville, Deep Learning (MIT Press, Cambridge, MA, 2016), http://www.deeplearningbook.org.

[2] Y. LeCun, Y. Bengio, and G. Hinton, Deep learning, Nature (London) 521, 436 (2015).

[3] D. Silver, A. Huang, C. J. Maddison, A. Guez, L. Sifre, G. Van Den Driessche, J. Schrittwieser, I. Antonoglou, V. Panneershelvam, M. Lanctot et al., Mastering the game of Go with deep neural networks and tree search, Nature (London) 529, 484 (2016).
[4] Y. D. Hezaveh, L. P. Levasseur, and P. J. Marshall, Fast automated analysis of strong gravitational lenses with convolutional neural networks, Nature (London) 548, 555 (2017).

[5] C. E. Petrillo et al., Finding strong gravitational lenses in the kilo degree survey with convolutional neural networks, Mon. Not. R. Astron. Soc. 472, 1129 (2017).

[6] N. Sun, J. Yi, P. Zhang, H. Shen, and H. Zhai, Deep learning topological invariants of band insulators, Phys. Rev. B 98, 085402 (2018).

[7] J. Carrasquilla and R. G. Melko, Machine learning phases of matter, Nat. Phys. 13, 431 (2017). 
[8] E. P. Van Nieuwenburg, Y.-H. Liu, and S. D. Huber, Learning phase transitions by confusion, Nat. Phys. 13, 435 (2017).

[9] P. Broecker, J. Carrasquilla, R. G. Melko, and S. Trebst, Machine learning quantum phases of matter beyond the fermion sign problem, Sci. Rep. 7, 8823 (2017).

[10] G. Carleo and M. Troyer, Solving the quantum many-body problem with artificial neural networks, Science 355, 602 (2017).

[11] X. Gao and L.-M. Duan, Efficient representation of quantum many-body states with deep neural networks, Nat. Commun. 8, 662 (2017)

[12] P. Baldi, P. Sadowski, and D. Whiteson, Searching for exotic particles in high-energy physics with deep learning, Nat. Commun. 5, 4308 (2014).

[13] P. Baldi, K. Cranmer, T. Faucett, P. Sadowski, and D. Whiteson, Parameterized neural networks for high-energy physics, Eur. Phys. J. C 76, 235 (2016).

[14] J. Cogan, M. Kagan, E. Strauss, and A. Schwarztman, Jetimages: Computer vision inspired techniques for jet tagging, J. High Energy Phys. 02 (2015) 118.

[15] P. Baldi, K. Bauer, C. Eng, P. Sadowski, and D. Whiteson, Jet substructure classification in high-energy physics with deep neural networks, Phys. Rev. D 93, 094034 (2016).

[16] A. Chakraborty, S. H. Lim, and M. M. Nojiri, Interpretable deep learning for two-prong jet classification with jet spectra, J. High Energy Phys. 07 (2019) 135.

[17] L.-G. Pang, K. Zhou, N. Su, H. Petersen, H. Stöcker, and X.-N. Wang, An equation-of-state-meter of quantum chromodynamics transition from deep learning, Nat. Commun. 9, 210 (2018).

[18] Y.-T. Chien, Probing heavy ion collisions using quark and gluon jet substructure with machine learning, Nucl. Phys. A 982, 619 (2019).

[19] J. Steinheimer, L.-G. Pang, K. Zhou, V. Koch, J. Randrup, and H. Stoecker, A machine learning study to identify spinodal clumping in high energy nuclear collisions, J. High Energy Phys. 12 (2019) 122.

[20] L.-G. Pang, K. Zhou, and X.-N. Wang, Interpretable deep learning for nuclear deformation in heavy ion collisions, Adv. Space Res. 63, 2 (2019).

[21] J. E. Bernhard, J. S. Moreland, S. A. Bass, J. Liu, and U. Heinz, Applying Bayesian parameter estimation to relativistic heavyion collisions: Simultaneous characterization of the initial state and quark-gluon plasma medium, Phys. Rev. C 94, 024907 (2016).

[22] J. E. Bernhard, J. S. Moreland, and S. A. Bass, Bayesian estimation of the specific shear and bulk viscosity of quark-gluon plasma, Nat. Phys. 15, 1113 (2019).

[23] D. Everett et al. (JETSCAPE), Phenomenological constraints on the transport properties of QCD matter with data-driven model averaging, Phys. Rev. Lett. 126, 242301 (2021).

[24] D. Everett et al. (JETSCAPE), Multisystem Bayesian constraints on the transport coefficients of QCD matter, Phys. Rev. C 103, 054904 (2021).

[25] K. Zhou, G. Endrődi, L.-G. Pang, and H. Stöcker, Regressive and generative neural networks for scalar field theory, Phys. Rev. D 100, 011501(R) (2019).

[26] R. S. Bhalerao, J.-Y. Ollitrault, S. Pal, and D. Teaney, Principal Component Analysis of Event-By-Event Fluctuations, Phys. Rev. Lett. 114, 152301 (2015).
[27] A. Mazeliauskas and D. Teaney, Subleading harmonic flows in hydrodynamic simulations of heavy ion collisions, Phys. Rev. C 91, 044902 (2015).

[28] A. M. Sirunyan, A. Tumasyan, W. Adam, F. Ambrogi, E. Asilar, T. Bergauer, J. Brandstetter, E. Brondolin, M. Dragicevic, J. Erö et al., Principal-component analysis of two-particle azimuthal correlations in $\mathrm{PbPb}$ and $p \mathrm{~Pb}$ collisions at CMS, Phys. Rev. C 96, 064902 (2017).

[29] Z. Liu, W. Zhao, and H. Song, Principal component analysis of collective flow in relativistic heavy-ion collisions, Eur. Phys. J. C 79, 870 (2019).

[30] Z. Liu, A. Behera, H. Song, and J. Jia, Robustness of principal component analysis of harmonic flow in heavy ion collisions, Phys. Rev. C 102, 024911 (2020).

[31] F. G. Gardim, F. Grassi, P. Ishida, M. Luzum, and J.-Y. Ollitrault, $p_{T}$-dependent particle number fluctuations from principal-component analyses in hydrodynamic simulations of heavy-ion collisions, Phys. Rev. C 100, 054905 (2019).

[32] I. Altsybeev, On feasibility of azimuthal flow studies with principal component analysis, J. Phys.: Conf. Ser. 1602, 012004 (2020).

[33] L. D. Landau and E. M. Lifshitz, Course of Theoretical Physics (Elsevier, Amsterdam, 2013).

[34] M. Gyulassy and L. McLerran, New forms of QCD matter discovered at RHIC, Nucl. Phys. A 750, 30 (2005).

[35] B. Müller and J. L. Nagle, Results from the relativistic heavy ion collider, Annu. Rev. Nucl. Part. Sci. 56, 93 (2006).

[36] P. Huovinen, Hydrodynamical description of collective flow, in Quark-Gluon Plasma 3 (World Scientific, Singapore, 2004), pp. 600-633.

[37] P. F. Kolb and U. Heinz, Hydrodynamic description of ultrarelativistic heavy-ion collisions, in Quark-Gluon Plasma 3 (Ref. [36]), pp. 634-714.

[38] U. Heinz and R. Snellings, Collective flow and viscosity in relativistic heavy-ion collisions, Annu. Rev. Nucl. Part. Sci. 63, 123 (2013).

[39] C. Gale, S. Jeon, and B. Schenke, Hydrodynamic modeling of heavy-ion collisions, Int. J. Mod. Phys. A 28, 1340011 (2013).

[40] H. Song, Y. Zhou, and K. Gajdošová, Collective flow and hydrodynamics in large and small systems at the LHC, Nucl. Sci. Technol. 28, 99 (2017).

[41] B. Muller, J. Schukraft, and B. Wyslouch, First results from $\mathrm{Pb}+\mathrm{Pb}$ collisions at the LHC, Annu. Rev. Nucl. Part. Sci. 62, 361 (2012).

[42] H. Song, S. A. Bass, U. Heinz, T. Hirano, and C. Shen, 200 A GeV Au+Au Collisions Serve a Nearly Perfect QuarkGluon Liquid, Phys. Rev. Lett. 106, 192301 (2011).

[43] B. Schenke, S. Jeon, and C. Gale, Elliptic and Triangular Flow in Event-By-Event (3+1)D Viscous Hydrodynamics, Phys. Rev. Lett. 106, 042301 (2011).

[44] C. Gale, S. Jeon, B. Schenke, P. Tribedy, and R. Venugopalan, Event-By-Event Anisotropic Flow in Heavy-Ion Collisions from Combined Yang-Mills and Viscous Fluid Dynamics, Phys. Rev. Lett. 110, 012302 (2013).

[45] X. Zhu, Y. Zhou, H. Xu, and H. Song, Correlations of flow harmonics in $2.76 A \mathrm{TeV} \mathrm{Pb}-\mathrm{Pb}$ collisions, Phys. Rev. C 95, 044902 (2017). 
[46] W. Zhao, H.-j. Xu, and H. Song, Collective flow in $2.76 \mathrm{TeV}$ and 5.02 $A \mathrm{TeV} \mathrm{Pb}+\mathrm{Pb}$ collisions, Eur. Phys. J. C 77, 645 (2017).

[47] C. Shen, Z. Qiu, H. Song, J. Bernhard, S. Bass, and U. Heinz, The iEBE-ISHNU code package for relativistic heavy-ion collisions, Comput. Phys. Commun. 199, 61 (2016).

[48] S. Pratt, E. Sangaline, P. Sorensen, and H. Wang, Constraining the Equation of State of Super-Hadronic Matter from Heavy-Ion Collisions, Phys. Rev. Lett. 114, 202301 (2015).

[49] H. Song and U. Heinz, Causal viscous hydrodynamics in $2+$ 1 dimensions for relativistic heavy-ion collisions, Phys. Rev. C 77, 064901 (2008).

[50] In the $(\tau, x, y, \eta)$ coordinate $\left(\tau=\sqrt{t^{2}-z^{2}}\right.$ and $\left.\eta=\frac{1}{2} \ln \frac{t+z}{t-z}\right)$, the energy density and pressure from $2+1 \mathrm{D}$ hydrodynamics are longitudinal boost-invariant without a dependence on $\eta, e=$ $e(\tau, x, y)$, and $p=p(\tau, x, y)$. Correspondingly, the four flow velocities are expressed as $\gamma_{\perp}\left[1, v^{x}(\tau, x, y), v^{y}(\tau, x, y), 0\right]$ with $v^{\eta}=0[36,37]$.

[51] M. L. Miller, K. Reygers, S. J. Sanders, and P. Steinberg, Glauber modeling in high energy nuclear collisions, Annu. Rev. Nucl. Part. Sci. 57, 205 (2007).

[52] T. Hirano and Y. Nara, Eccentricity fluctuation effects on elliptic flow in relativistic heavy ion collisions, Phys. Rev. C 79, 064904 (2009).

[53] H.-J. Drescher and Y. Nara, Effects of fluctuations on the initial eccentricity from the color glass condensate in heavy ion collisions, Phys. Rev. C 75, 034905 (2007).

[54] H.-J. Drescher and Y. Nara, Eccentricity fluctuations from the color glass condensate at RHIC and LHC, Phys. Rev. C 76, 041903(R) (2007).

[55] L. Pang, Q. Wang, and X.-N. Wang, Effects of initial flow velocity fluctuation in event-by-event $(3+1) \mathrm{D}$ hydrodynamics, Phys. Rev. C 86, 024911 (2012).
[56] H.-j. Xu, Z. Li, and H. Song, High-order flow harmonics of identified hadrons in $2.76 A \mathrm{TeV} \mathrm{Pb}+\mathrm{Pb}$ collisions, Phys. Rev. C 93, 064905 (2016).

[57] W. Zhao, Y. Zhou, H. Xu, W. Deng, and H. Song, Hydrodynamic collectivity in proton-proton collisions at $13 \mathrm{TeV}$, Phys. Lett. B 780, 495 (2018).

[58] J. S. Moreland, J. E. Bernhard, and S. A. Bass, Alternative ansatz to wounded nucleon and binary collision scaling in highenergy nuclear collisions, Phys. Rev. C 92, 011901(R) (2015).

[59] K. He, X. Zhang, S. Ren, and J. Sun, Deep residual learning for image recognition, in Proceedings of the IEEE Conference on Computer Vision and Pattern Recognition (IEEE, New York, 2016), pp. 770-778.

[60] H. Song, Causal viscous hydrodynamics for relativistic heavy ion collisions, Ph.D thesis, The Ohio State University, 2009, arXiv:0908.3656.

[61] L.-G. Pang, H. Petersen, and X.-N. Wang, Pseudorapidity distribution and decorrelation of anisotropic flow within the open-computing-language implementation CLVisc hydrodynamics, Phys. Rev. C 97, 064918 (2018).

[62] D. Bazow, U. W. Heinz, and M. Strickland, Massively parallel simulations of relativistic fluid dynamics on graphics processing units with CUDA, Comput. Phys. Commun. 225, 92 (2018).

[63] P. Zhang, H. Shen, and H. Zhai, Machine Learning Topological Invariants with Neural Networks, Phys. Rev. Lett. 120, 066401 (2018).

[64] W. Luo, Y. Li, R. Urtasun, and R. Zemel, in Advances in Neural Information Processing Systems (MIT Press, Cambridge, U.K., 2016), pp. 4898-4906.

[65] M. Raissi, P. Perdikaris, and G. E. Karniadakis, Physicsinformed neural networks: A deep learning framework for solving forward and inverse problems involving nonlinear partial differential equations, J. Comput. Phys. 378, 686 (2019). 